\title{
Causas de insônia nos primeiros anos de vida e repercussão nas mães: atualização
}

\author{
Causes of insomnia in the first years of life and maternal consequences: an update
}

\author{
Eduardina Tenenbojm¹, Sueli Rossini', Eduard Estivill ${ }^{2}$, Francisco Segarra ${ }^{3}$, Rubens Reimão ${ }^{4}$
}

\section{RESUMO}

Objetivo: Revisar a literatura sobre insônia de crianças pequenas e seus aspectos relacionados às repercussões familiares, à abordagem pediátrica e ao seu tratamento.

Fontes de dados: Levantamento de publicações indexadas no Medline e Lilacs entre os anos de 1998 e 2008, rastreadas com a combinação dos descritores: "sleep", "insomnia", "child", "depression”, "mother-child relationship", além de teses e capítulos de livros pertinentes ao assunto.

Síntese dos dados: A insônia da criança pequena, definida como dificuldade repetida em iniciar e/ou manter o sono, é queixa frequente na clínica pediátrica e usualmente traz repercussões nos pais relativas à privação de sono. Aos dois a três meses de idade, há condições biológicas para consolidação das horas de sono à noite; estando a conduta parental associada às dificuldades neste processo. Estudos demonstram que há associação entre insônia do bebê e depressão nas mães, e a insônia pode ser manifestação de dificuldades no desenvolvimento psíquico da criança pequena, que acontece na relação com sua mãe.

Conclusões: $\mathrm{O}$ ritmo de sono nos bebês pode e deve estabelecer-se precocemente; os hábitos para o sono devem basear-se nas medidas de higiene do sono. A depressão nas mães, que pode contribuir para a insônia do bebê, idealmente poderia ser detectada pelo pediatra, prevenindo esse comprometimento das mães e de seus bebês.

Palavras-chave: sono; distúrbios do início e manutenção do sono; criança; depressão; relações mãe-filho.

Instituição: Hospital das Clínicas da Faculdade de Medicina da Universidade de São Paulo (USP), São Paulo, SP, Brasil

'Doutora; Pesquisadora do Grupo de Pesquisa Avançada em Medicina do Sono do Hospital das Clínicas da Faculdade de Medicina da USP, São Paulo, SP, Brasil

${ }^{2}$ Doutor; Diretor da Clinica del Sueño Estivill do Institut Universitari Dexeux, Barcelona, Espanha

${ }^{3}$ Doutor; Clinica del Sueño Estivill do Institut Universitari Dexeux, Barcelona, Espanha

${ }^{4}$ Livre Docente; Professora da Divisão de Clínica Neurológica do Hospital das Clínicas da Faculdade de Medicina da USP; Líder do Grupo de Pesquisa Avançada em Medicina do Sono do Hospital das Clínicas da Faculdade de Medicina da USP, São Paulo, SP, Brasil

\section{ABSTRACT}

Objective: To review the literature on insomnia in small children, its aspects related to effects on parents, pediatric approach and treatment.

Data sources: A survey of publications indexed in Medline and Lilacs between the years 1998 and 2008, searched through the combination of the descriptors "sleep", "insomnia”, "child”, "depression", "mother-child relationship", besides thesis and chapters of books concerning the subject.

Data synthesis: The small child insomnia, defined as repeated difficulty in initiating and/or maintaining sleep, is a common complaint in the pediatric clinic, and usually entails repercussions on parents related to sleep deprivation. At about two to three months of age, there are already biological conditions for consolidating sleep hours during the night. Parental behavior is associated to difficulties to establish this process. Studies point out that baby's insomnia and mother's depression are closely related. Probably, the insomnia is a sign of the difficulties in the small child's psychic development in the context of mother-child relationship.

Conclusions: Rhythmic sleep in babies can and should be established early in life; sleep habits should be built on rules of sleep hygiene. Mother's depression, which may contribute to baby's insomnia, should ideally be detected by pediatricians, thus preventing such suffering for mothers and babies.

Key-words: sleep; sleep initiation and maintenance disorders; child; depression; mother-child relations.

\author{
Endereço para correspondência: \\ Eduardina Tenenbojm \\ Rua das Bandeiras, 72, conj. 34 - Jardim \\ CEP: 09090-780 - Santo André/SP \\ E-mail: eduardina.t@uol.com.br \\ Fonte financiadora: Coordenação de Pessoal de Nível Superior (Capes), \\ processo n. ${ }^{\circ} 2199 / 07-6$
}

Conflitos de interesse: nada a declarar

Recebido em: 6/2/09

Aprovado em: 3/5/09 


\section{Introdução}

A insônia da criança pequena, definida como dificuldade repetida em iniciar e/ou manter o sono, é caracterizada por grande número de despertares durante a noite, ocasionalmente com dificuldade em reiniciar o sono, sendo queixa frequente na clínica pediátrica. Na maioria das vezes, se manifesta após ter provocado repercussões e dificuldades nos pais e na rotina familiar relativas à privação de sono.

\section{Objetivo}

Esse estudo visou revisar a literatura por meio de levantamento de publicações indexadas no Medline e Lilacs, no período de 1998 a 2008, rastreadas pela combinação dos descritores: "sleep”, "insomnia", "child", "depression", "mother-child relationship", além de teses e capítulos de livros pertinentes ao assunto.

\section{A insônia da criança pequena na Classificação Internacional dos Distúrbios do Sono}

De acordo com a Classificação Internacional de Distúrbios de Sono, segundo revisão realizada em 2005, a insônia das crianças pequenas pode apresentar-se como insônia comportamental da infância, que ocorre entre 10 e $30 \%$ da população infantil ${ }^{(1)}$. O diagnóstico baseia-se nos sintomas da criança referidos pela mãe. A insônia comportamental da infância apresenta-se sob dois tipos:

1. Insônia de associação para iniciar o sono: quando, para adormecer, a criança requer condições especiais, como algum procedimento específico dos pais (embalar por longo tempo, passear de carro, entre outras), que, pela repetição, terminam por demandar alto investimento dos pais. Na ausência desse fator associado, há prejuízo no sono, com atraso tanto ao iniciar quanto para retomar o sono quando interrompido.

2. Insônia por dificuldades de imposição de limites: quando há recusa ou protelação por parte da criança em ir para a cama. O cuidador impõe poucos limites ou limites inconsistentes para a criança e, por vezes, há queixas associadas de problemas de comportamento durante o dia.

$\mathrm{Na}$ criança pequena, pode ocorrer insônia por higiene do sono inadequada, em geral associada a hábitos impróprios, como excesso de estimulação física, mental ou emocional próximo ao horário de recolher-se para dormir e falha no estabelecimento de horário e de rituais para o adormecer.

\section{Desenvolvimento de padrões de sono nos primeiros meses de vida}

No recém-nascido, há três tipos reconhecidos de sono: sono ativo, precursor do sono em que acontecem os movimentos rápidos dos olhos (REM); sono quieto, precursor do sono nãoREM (NREM) ${ }^{\text {a }}$; e sono indeterminado, que não apresenta as características polissonográficas do sono quieto nem as do sono ativo. O sono quieto é caracterizado por leves movimentos musculares e ciclos respiratórios rítmicos. Durante o sono ativo, acontecem movimentos de sucção, sorrisos, tremores, franzimento de sobrancelhas, respiração irregular e movimentos de membros. Nas idades posteriores, o sono REM caracteriza-se por perda completa ou diminuição do tônus muscular, o oposto do que se percebe no recém-nascido. Com o amadurecimento do recém-nascido, o sono indeterminado torna-se bem definido em sono REM ou sono NREM. Comparada à arquitetura do sono de crianças de mais idade, o neonato apresenta diferenças: nas primeiras semanas de vida, o estágio de sono ativo prepondera, com 50 a $80 \%$ do tempo total de sono; o ciclo de sono dura de 50 a 60 minutos e o início ocorre no sono ativo ${ }^{(2)}$. Cada período de sono tem de um a dois ciclos, de modo que a criança acorda com mais frequência. Em geral, esses períodos estão acoplados aos ciclos de fome. Aos seis meses de idade, o início do sono se dá no sono NREM e a propensão ao movimento, durante o sono REM, é substituída pela típica paralisia muscular que caracteriza esse estágio de sono no adulto.

$\mathrm{O}$ fato de o recém-nascido passar grande parte do dia adormecido faz pensar que o sono tenha papel importante no desenvolvimento orgânico e mental no início da vida. O tempo total de sono diário, com o aumento da idade, reduz-se paulatinamente.

Até os dois a três meses, o ritmo circadiano se estabelece e a criança passa a apresentar diminuição do sono diurno e consolidação das horas de sono à noite. Nessa idade, há condições biológicas de estabelecimento do ritmo circadiano e as crianças tornam-se mais sensíveis ao meio ambiente, respondendo ao claro/escuro para organizar seu ritmo vigília/sono. O ritmo da casa e as rotinas noturnas passam a funcionar como sinais sociais ou ambientais, com influência no ritmo vigília/sono do bebê. Como consequência, consolidam-se as horas de sono no período noturno ${ }^{(3)}$. Em seguida, a criança começa a ter longos períodos 
de vigília durante o dia e a proporção de sono REM começa a decrescer para 30 ou $40 \%$ do sono total do $\operatorname{dia}^{(4)}$. No primeiro ano de vida, as características do ciclo sono-vigília traduzem a maturação do sistema nervoso central ${ }^{(2)}$. Há grande faixa de variação do sono normal nos primeiros anos de vida. Padrões de sono normal da criança podem tornar-se motivo de queixa, se a rotina da família sofrer grande interferência ${ }^{(5)}$.

Jenni $e t a l^{(6)}$, em estudo sobre o sono em crianças de um a dez anos de idade, concluíram que a quantidade de sono diminui com a idade e que, no desenvolvimento da criança, não há alterações que possam ser utilizadas como marcos da maturação do sono infantil. Afirmam também que, aos 12 meses, 96\% das crianças dormem entre 11 horas 40 minutos e 16 horas 50 minutos por dia, e que os pais devem ser orientados sobre as características individuais no sono das crianças, visto que tanto pode haver dormidores longos (aqueles que necessitam de maior número de horas dormidas por dia) quanto dormidores curtos.

Touchette et $a l^{(7)}$ avaliaram fatores relacionados à fragmentação do sono em 1.741 crianças entre cinco e 29 meses e concluíram que a consolidação do sono desenvolve-se rapidamente na infância precoce; a conduta parental nas rotinas para adormecer e a resposta aos despertares noturnos estão firmemente associados à consolidação das horas de sono à noite; a alimentação noturna é fator fortemente associado à fragmentação do sono entre as crianças de cinco meses de idade; a presença dos pais para adormecer está associada às dificuldades de sono nas crianças com idade entre um ano e cinco meses e dois anos e cinco meses.

Clinicamente, as cólicas do bebê muitas vezes aparecem associadas aos distúrbios de sono, sendo que o comportamento dos pais para diminuir o choro do bebê (por exemplo, embalar com frequência, passear de carro, entre outras) levam à adoção de condutas que não favorecem o padrão de sono normal ${ }^{(8,9)}$.

Os hábitos e rituais para o sono são importantes, mas é preciso considerar as variações culturais. Em estudo realizado em Barcelona, Espanha, os autores observaram que, dentre os bebês insones, foi frequente as crianças necessitarem da presença de um dos pais para adormecer, porém não foi habitual os pais alimentarem os bebês durante a noite ${ }^{(10)}$. Já estudo australiano com 156 mães de crianças com insônia grave, com idade entre seis e 12 meses, detectou que as crianças que sofriam de insônia dormiam mais frequentemente na cama dos pais, eram embaladas para adormecer, demorando mais tempo para dormir e despertando com maior frequência e por maiores períodos durante a noite ${ }^{(11)}$.

Assim, deve-se considerar o amplo leque de alterações maturacionais normais, tanto físicas como do desenvolvimento na infância, e ainda as influências culturais, ambientais e sociais na definição do sono adequado para a criança pequena ${ }^{(12)}$.

\section{O ciclo circadiano}

O núcleo supraquiasmático no hipotálamo funciona como um relógio biológico que regula o ritmo circadiano em mamíferos, recebendo informação visual direta relativa à luminosidade. A integração desse sistema neuroendócrino é mediada pela melatonina - hormônio secretado pela glândula pineal - que aumenta a tendência ao sono e indica ao cérebro o conceito de noite, escuridão ${ }^{(13)}$.

O ciclo circadiano é gerado endogenamente com um período de duração de aproximadamente 24 horas. Há evidências de que o sistema circadiano desenvolve-se desde o período pré-natal e de que, após o nascimento, mature progressivamente. O sistema circadiano de primatas jovens é responsivo à luz em estágios muito prematuros e a baixa intensidade de luminosidade pode sensibilizar o relógio biológico. No homem, com cerca de 12 semanas de vida, é possível detectar o ritmo dia-noite de produção de melatonina ${ }^{(14)}$.

No organismo, o precursor da melatonina é o aminoácido triptofano e seu metabólito excretado pela urina é a 6-sulfatoximelatonina. Há relação temporal entre o ritmo de excreção da 6-sulfatoximelatonina na urina de bebês alimentados exclusivamente ao seio materno e o triptofano dosado no leite da mãe, o que aponta a ação do leite materno como facilitador do sono dos bebês e de sua ritmicidade ${ }^{(15)}$.

\section{A queixa de insônia nos consultórios de pediatria e a conduta dos pediatras}

Estudos mostram que as queixas da mãe em relação ao sono dos seus bebês são consistentes, principalmente no que se refere ao horário de dormir e de despertar e à quantidade e duração do sono noturno ${ }^{(16)}$.

As famílias costumam ter no pediatra a maior fonte de conhecimento sobre o comportamento e desenvolvimento das crianças. Entretanto, muitos pediatras revelam falhas em seu treinamento a esse respeito e baseiam suas orientações amplamente nos próprios valores culturais, em interação com sua experiência clínica e pessoal ${ }^{(5)}$. Ao avaliar a queixa de distúrbio de sono de uma criança, deve-se levar em conta o ambiente em que ela vive e os valores da cultura de seus pais, além das características biológicas de cada criança.

Por ser a insônia das crianças pequenas motivo repetido de queixa, com ampla faixa de variação do sono normal para cada 
idade, não-passível de medicação sob protocolo seguro, passa ela a ser subavaliada, subdiagnosticada e torna-se fator de angústia profissional para o médico pediatra, que acaba por lançar mão de sua experiência pessoal para orientar os pacientes.

Estudo em que foram observadas 799 crianças do nascimento até os 36 meses apontaram que um padrão persistente de despertares noturnos frequentes parecia estar relacionado a risco de ferimentos e lesões corporais não-intencionais ${ }^{(17)}$. Os autores esclarecem que tais lesões podem decorrer da fadiga e do cansaço dessas crianças, fator que as torna menos atentas, levando-as a comportamentos mais arriscados. Por outro lado, os pais, também cansados e fatigados por dormirem mal durante a noite, supervisionam as crianças com menos atenção. Esses aspectos devem ser levados em conta pelos profissionais de saúde no atendimento às crianças.

\section{Distúrbios de sono de origem orgânica em crianças pequenas}

Algumas condições clínicas alteram o padrão de sono das crianças pequenas:

- Doenças agudas, como otites, distúrbios respiratórios ou outras que cursem com dor e/ou desconforto. Costumam afetar a criança por tempo limitado, durante o período da doença. Quando diagnosticadas e tratadas, contribuem ao alívio do distúrbio de sono.

- Alergia ao leite de vaca, causando despertares frequentes, com ciclos de sono curtos e aumento do estágio $1 \mathrm{NREM}^{(18)}$.

- Doença do refluxo gastroesofágico, que costuma acordar a criança por dor, usualmente após a terceira hora de sono. A dor é aliviada quando se retira a criança do berço ${ }^{(19)}$.

- Doenças neurológicas, nas quais o distúrbio de sono aparece como manifestação secundária ${ }^{(20)}$.

\section{Tratamento da insônia da criança pequena}

\section{Tratamento medicamentoso}

Idiazábal e Estivill analisaram os medicamentos utilizados em insônia infantil em 267 crianças entre seis meses e cinco anos, verificando que $43 \%$ dos pacientes haviam sido tratados farmacologicamente ${ }^{(21)}$. Os fármacos utilizados foram neurolépticos, anti-histamínicos, barbitúricos, benzodiazepínicos e ansiolíticos, tendo o tratamento durado 8,7 dias, em média. A eficácia foi considerada nula pelos pais em $95 \%$ dos casos, sendo eficaz nos $5 \%$ restantes nos primeiros dias de uso, reaparecendo a sintomatologia mesmo com a continuação do tratamento.
Em termos experimentais, a melatonina sintetizada para uso por via oral é uma opção medicamentosa para a insônia do adulto e vem sendo estudada em crianças sadias ou com distúrbios neurológicos. No Brasil, seu uso não é permitido. Seu efeito baseia-se na diminuição da latência do sono e melhora na eficiência e no tempo total de sono. Seu uso na clínica pediátrica demanda, para o futuro, maiores investigações que assegurem eficácia e segurança de prescrição ${ }^{(21-25)}$.

Mindell et al ${ }^{(26)}$ relatam que o tratamento medicamentoso da insônia na Pediatria é uma necessidade médica ainda não encontrada. É preciso desenvolver diretrizes rigorosas de manejo farmacológico da insônia nessa faixa etária e é vital informar ao médico clínico sobre a segurança e a eficácia de sua prescrição.

\section{Abordagens comportamentais da insônia das crianças}

Baseiam-se no restabelecimento do ritmo sono-vigília com o treinamento dos pais, que utilizam medidas comportamentais como sincronizadores externos do sono da criança ou intervenções para a correção do ritmo diário de sono ${ }^{(27)}$. Tais abordagens consistem no suporte aos pais para informá-los sobre a capacidade de o bebê autoconfortar-se e na educação dos pais com respeito às características de desenvolvimento de seus filhos ${ }^{(28)}$.

O estudo de revisão de 52 artigos realizado por Mindell et a ${ }^{(29)}$ sobre tratamento para problemas de sono em crianças pequenas observou que a terapia comportamental produz mudanças duráveis e confiáveis e enfatizou a necessidade de mais pesquisas sobre o sono em Pediatria para padronizar critérios diagnósticos e medidas objetivas, inclusive os agentes farmacológicos.

\section{Aspectos psíquicos relacionados ao sono}

Segundo Freud, os sonhos são um modo de contato com os conteúdos psíquicos. Melanie Klein postula existir intensa atividade mental nos bebês nos primeiros meses de vida, o que coincide temporalmente com a época em que passam a maior parte do tempo dormindo ${ }^{(30,31)}$. O fato de o recém-nascido passar grande parte do tempo total de sono no estágio de sono ativo - precursor do sono REM, quando acontece o maior número de sonhos - pode sugerir que o sono contribua para o desenvolvimento psíquico do bebê.

O desenvolvimento biológico e das relações psíquicas ocorre simultaneamente na criança pequena; quanto ao desenvolvimento das relações psíquicas, denominadas relações de objeto, elas acontecem principalmente na relação com sua mãe. O estabelecimento do ciclo sono-vigília é um indicador das primeiras fases do desenvolvimento psíquico. Segundo McDougall ${ }^{(32)}$, 
"podemos descobrir o protótipo mais antigo da atividade psíquica na capacidade que a criança tem de adormecer e permanecer adormecida" (p.88).

A relação inicial do bebê com sua mãe foi tematizada nos estudos psicanalíticos, buscando compreender o desenvolvimento psíquico no início da vida. Na relação mãe-bebê, a função alfa é a capacidade de transformação dos elementosbeta, que se referem a sensações e experiências emocionais primitivas, extremamente desconfortáveis ao bebê, em elementos-alfa ${ }^{(33)}$. No início da vida, a função alfa é exercida pela mãe, cujo papel consiste em ser "um continente adequado, de modo a acolher, conter, decodificar e devolver para o filho aquilo que ele projetou nela, agora desintoxicado, significado e nomeado", que se constituirá em elementos-alfa ${ }^{(34)}$. Essa capacidade da mãe de transformar os elementos-beta em elementos-alfa denomina-se rêverie ${ }^{\mathrm{b}}$. O armazenamento desses elementos-alfa enseja no indivíduo o pensar e o aprender com as experiências ${ }^{(33)}$.

Segundo Melanie Klein, por meio da projeção e introjeção na relação com a mãe, o bebê estabelecerá firmemente objetos internos bons (uma imagem interna da mãe), que o permita separar-se dela para adormecer, conciliando o sono após as mamadas ${ }^{(31)}$. No caso da criança com insônia, por alguma falha no mecanismo de comunicação mãe-bebê, esse é incapaz de internalizar sua mãe enquanto guardiã de seu sono ${ }^{(32)}$. Esses bebês agitados buscam incessantemente no mundo exterior uma fonte de tranquilidade que deveria provir de sua relação com a mãe por meio do estabelecimento do objeto bom.

\section{As mães, seus bebês insones e a depressão}

Segundo o Manual de Diagnóstico dos Distúrbios Mentais, quarta edição revisada da Associação Americana de Psiquiatria, e o Código Internacional de Doenças, décima revisão (CID-10) da Organização Mundial de Saúde (OMS), sob o código F32, a depressão pode apresentar-se com diferentes graus: leve, moderada ou grave $\mathrm{g}^{(36,37)}$.

A depressão pós-parto ou puerperal tem a especificidade de ocorrer imediatamente após ou em até poucas semanas (quatro semanas) após o parto. Inicialmente, era considerada, para diagnóstico, distinta de outras doenças psiquiátricas. Estudos posteriores mostraram que a depressão puerperal é clinicamente indistinguível da depressão que pode ocorrer em outras épocas da vida da mulher ${ }^{(36)}$.
Na relação mãe-bebê, se a mãe estiver ocupada psiquicamente com seus objetos perdidos (o que se traduziria clinicamente pela depressão), não conseguirá investir na relação com seu bebê. Queixas de distúrbios de sono dos bebês associadas a sintomas de depressão materna são comuns. Há forte associação entre os dois, mas, se a mãe dorme bem, essa relação se atenua ${ }^{(38)}$. A qualidade de sono da criança e a qualidade de sono da mãe estão associadas; a má qualidade de sono das mães está associada a distúrbios de humor, estresse e fadiga ${ }^{(39)}$. Por outro lado, nas mães portadoras de depressão cujos filhos apresentam distúrbios do sono, o tratamento do distúrbio do sono da criança exerce papel significante na melhora da saúde emocional da mãe e dos laços entre a mãe e o bebêt(40).

O Estudo Longitudinal das Crianças Australianas, que analisou 5.107 indivíduos, indicou que os distúrbios de sono nas crianças pequenas e nos pré-escolares estão associados a prejuízos na saúde tanto das mães quanto dos pais, de modo especial na saúde mental das mães sem antecedentes clínicos de depressão ${ }^{(41)}$.

Tenenbojm ${ }^{(42)}$, estudando insônia de crianças pequenas e suas relações com depressão materna, em estudo inicial, concluiu que são mais frequentes os sinais e sintomas depressivos em mães de bebês com insônia e que essas mães mais frequentemente alimentam seus bebês durante a noite. Encontrou também, entre as mães dos bebês insones, algumas com ideação suicida.

\section{Considerações finais}

A insônia dos bebês é uma condição clínica que necessita ser avaliada sob vários aspectos. $\mathrm{O}$ ritmo de sono nos bebês pode e deve estabelecer-se precocemente. Os hábitos para o sono devem basear-se nas medidas de higiene do sono:

- Estabelecer horário, rotinas e rituais consistentes para o sono.

- Evitar estimulação física, mental ou emocional perto da hora de dormir.

- Evitar oferecer alimentação durante a noite.

- Evitar dormir com alguma fonte luminosa durante toda a noite.

- É desejável que a criança habitue-se a adormecer sozinha, sem a presença do cuidador.

Há associação de insônia nos bebês e depressão nas mães. No futuro seria importante desenvolver mecanis-

b O conceito de rêverie é parte integrante da teoria do pensamento de Bion e guarda relações com a capacidade de tolerar frustrações, a necessidade de amor e com o processo do conhecimento. Bion a define assim: "Quando a mãe ama seu bebê, o que ela faz com isto?" E responde: "Além dos canais físicos de comunicação, minha impressão é de que seu amor é expresso pela rêverie. Tradução livre da pesquisadora (Bion citado por Sandler(35) p. 645$)$. 
mos de os pediatras levantarem suspeita de depressão nas mães com encaminhamento para tratamento médico e/ou psicológico, o que poderia ter caráter preventivo em relação a esse comprometimento das mães e de seus bebês.

\section{Referências bibliográficas}

1. American Academy of Sleep Medicine. ICSD-2 - The international classification of sleep disorders - diagnostic and coding manual. 2nd ed. Westchester: American Academy of Sleep Medicine; 2005.

2. Reimão R. Sono normal na infância. In: Diament AJ, Cypel S, editores. Neurologia infantil. 4a ed. São Paulo: Atheneu; 2005. p. 1335-44.

3. Sheldon SH. Sleep in infants and children. In: Lee-Chiong Jr TL, Sateia M, Carskadon MA, editors. Sleep medicine. Philadelphia: Hanley \& Belfus; 2002. p. 99-103.

4. Roffwarg HP, Muzio JN, Dement WC. Ontogenetic Development of the Human Sleep-Dream Cycle. Science 1966;152:604-19.

5. Jenni OG, O'Connor BB. Children's sleep: an interplay between culture and biology. Pediatrics 2005;115:204-16.

6. Jenni OG, Molinari L, Caflisch JA, Largo RH. Sleep duration from ages 1 to 10 years: variability and stability in comparison with growth. Pediatrics 2007;120:e769-76

7. Touchette E, Petit D, Paquet J, Boivin M, Japel C, Tremblay RE et al. Factors associated with fragmented sleep at night across early childhood. Arch Pediatr Adolesc Med 2005;159:242-9.

8. Thiedke CC. Sleep disorders and sleep problems in childhood. Am Fam Physician 2001;63:277-84.

9. Smart J, Hiscock H. Early infant crying and sleeping problems: a pilot study of impact on parental well-being and parent-endorsed strategies for management. J Paediatr Child Health 2007;43:284-90.

10. Tenenbojm E, Angelis G, Rossin S, Estivill E, Segarra F, Reimão R. Insomniac children maternal sleep and mood in São Paulo and Barcelona. Arq Neuropsiquiatr 2008;66:482-4.

11. Hiscock H, Wake M. Randomised controlled trial of behavioural infant sleep intervention to improve infant sleep and maternal mood. BJM 2002;324:1062-5.

12. Owens J. Classification and epidemiology of childhood sleep disorders. Prim Care 2008:35:533-46.

13. Velluti RA. Fisiologia do sono. In: Reimão R, editor. Sono: estudo abrangente. 2a ed. São Paulo: Atheneu; 1996. p. 1-15.

14. Rivkees SA. Developing circadian rhythmicity in infants. Pediatr Endocrinol Rev 2003;1:38-45.

15. Cubero J, Valero V, Sánchez J, Rivero M, Parvez $H$, Rodríguez $A B$ et al. The circadian rhythm of tryptophan in breast milk affects the rhythms of 6-sulfatoxymelatonin and sleep in newborn. Neuro Endocrinol Lett 2005;26:657-61.

16. Acebo C, Sadeh A, Seifer R, Tzischinsky O, Hafer A, Carskadon MA. Sleep/ wake patterns derived from activity monitoring and maternal report for healthy 1-to 5-years-old children. Sleep 2005;28:1568-77.

17. Schwebel DC, Brezausek CM. Nocturnal awakenings and pediatric injury risk. J Pediatr Psychol 2008;33:323-32.

18. Kahn A, François G, Sottiaux M, Rebuffat E, Nduwimana M, Mozin MJ et al. Sleep characteristics in milk-intolerant infants. Sleep 1988;11:291-7.

19. Madeira IR, Aquino LA. Difficulties in the management of sleep and eating disorders. J Pediatr (Rio J) 2003;79:S43-54.

20. Kohrman MH, Carney PR. Sleep-related disorders in neurologic disease during childhood. Pediatr Neurol 2000;23:107-13.

\section{Agradecimentos}

Agradecemos o apoio recebido da Coordenação de Aperfeiçoamento de Pessoal de Nível Superior (Capes), sob a forma de bolsa de estudos.

21. Idiazábal MA, Estivill ES. Tratamiento del insomnio en niños: aspectos farmacológicos. An Pediatr (Barc) 2003;59:239-45.

22. Ross C, Davies P, Whitehouse W. Melatonin treatment for sleep disorders in children with neurodevelopmental disorders: an observational study. Dev Med Child Neurol 2002;44:339-44.

23. Reed MD, Findling RL. Overview of current management of sleep disturbances in children: I-Pharmacotherapy. Curr Ther Res Clin Exp 2002;63:B18-37.

24. Younus M, Labellarte MJ. Insomnia in children: when are hypnotics indicated? Paediatr Drugs 2002;4:391-403.

25. Nunes ML, Cavalcante V. Clinical evaluation and treatment of insomnia in childhood. J Pediatr (Rio J) 2005;81:277-86.

26. Mindell JA, Emslie G, Blumer J, Genel M, Glaze D, Ivanenko A et al Pharmacologic management of insomnia in children and adolescents: consensus statement. Pediatrics 2006;117:1223-32.

27. Estivill E. Childhood insomnia due to disorderly habits. Rev Neurol 2000;30: 188-91.

28. Skuladottir A, Thome M. Changes in infant sleep problems after a familycentered intervention. Pediatr Nurs 2003;29:375-8.

29. Mindell JA, Kuhn B, Lewin DS, Meltzer LJ, Sadeh A; American Academy of Sleep Medicine. Behavioral treatment of bedtime problems and night wakings in infants and young children. Sleep 2006;29:1263-76.

30. Freud S. Obras psicológicas completas de Sigmund Freud. Edição Standard Brasileira. 2a ed. Rio de Janeiro: Imago; 1987.

31. Klein M. Inveja e gratidão e outros trabalhos. Rio de Janeiro: Imago; 1991.

32. McDougall J. Teatros do corpo: o psicossoma em psicanálise. São Paulo: Martins Fontes; 1996.

33. Bion W. Elementos de psicanálise. Rio de Janeiro: Zahar; 1962

34. Zimerman DE. Vocabulário contemporâneo de psicanálise. São Paulo: Artmed; 2001.

35. Sandler PC. The language of Bion: a dictionary of concepts. London: Karnac Books; 2005.

36. Associação Americana de Psiquiatria. DSM-IV Manual diagnóstico e estatístico de transtornos mentais. 4a ed rev. Porto Alegre: Artmed; 2002.

37. Organização Mundial da Saúde. Classificação internacional de doenças e problemas relacionados à saúde - CID-10. 10a ed. São Paulo: Edusp; 1998.

38. Hiscock H, Wake M. Infant sleep problems and postnatal depression: a community-based study. Pediatrics 2001;107:1317-22.

39. Meltzer LJ, Mindell JA. Relationship between child sleep disturbances and maternal sleep, mood, and parenting stress: a pilot study. J Fam Psychol 2007;21:67-73.

40. Matthey S, Speyer J. Changes in unsettled infant sleep and maternal mood following admission to a parentcraft residential unit. Early Hum Dev 2008;84:623-9.

41. Martin J, Hiscock H, Hardy P, Davey B, Wake M. Adverse associations of infant and child sleep problems and parent health: an Australian population study. Pediatrics 2007;119:947-55.

42. Tenenbojm E. Eficácia adaptativa, padrão de sono e depressão em mães de crianças de três a 24 meses com insônia [tese de doutorado]. São Paulo (SP): USP; 2008. 